\title{
Principles of Cryometric Impurity Determination as Applied to Samples of Small Sizes
}

\author{
Charles P. Saylor and Gaylon S. Ross
}

(October 21, 1963)

\begin{abstract}
A consideration of the factors that tend to prevent attainment of thermal and thermodynamic ?equilibrium during thermometric methods of purity analysis suggests that the problems largely originate from spontaneously introduced inequalities in composition. During either a freezing or melting cycle, the solid phase settles in the liquid. This results in a greater average purity in the bottom than in the upper part of the vessel. The effects would be particularly strong during a melting sequence where the liquid released by melting of the solid would provide the composition al environment for the establishment of final temperature. This hypothesis has been tested by the design of a new cell for small samples. The design incorporates use of small gold pans to hold solid and liquid in close contact. The results are comparable in precision to those from conventional time-temperature curves with much larger samples, a condition not ordinarily possible with small samples.
\end{abstract}

\section{Introduction}

Cryometric techniques capable of accurate determinations of purity have been developed. When samples as large as 40 to $100 \mathrm{ml}$ are used, the procedures available at the present time reduce successfully the errors resulting from imperfect attainment of equilibrium, both thermal and thermodynamic. These errors become progressively larger, however, with decreasing size of the sample. It was our purpose in this investigation to analyze the errors that persist in the ordinary large scale determinations of purity and also to alter the procedures in such a way that sufficient accuracy could be attained even with drastically reduced samples.

\section{Principles}

When a system is surrounded by, but relatively well-insulated from, a constant-temperature environment, the rate of loss or gain of heat by the system will be essentially constant, providing the difference in temperature between the system and the environment is very large with respect to the temperature change taking place within the test system. If there are no chemical reactions or phase transitions, the temperature of the system will change at a rate inversely proportional to the product of the specific heat of the system and the mass. In cryometry the system consists of a sample contained in a closed cell. As long as the sample is completely liquified, or fully solidified, the above relationship holds. However, when freezing or melting begins, the rate of change in temperature will be reduced because of the latent heat of the sample.

As long as compositional, thermal, and thermodynamic equilibrium are maintained and nothing occurs to disturb the uniform loss of heat from the sample container to its environment, the curve of temperature as a function of time during a freezing or melting sequence will be close to the hyperbolic form. One asymptote of this hyperbola will correspond to the melting point of the pure substance, and the other asymptote will represent the cooling rate of the solidified sample and the cell. A slight imperfection in the hyperbola is caused by the fact that the specific heat contribution during freezing is influenced proportionally by the specific heat of both the portion of the sample that is liquid and the portion that is solid. When the form of this hyperbola has been established, the intersection of the curve with the liquid line gives the melting point of the actual sample. The difference between freezing temperature of the sample and the calculated freezing point of the pure material is proportional to the mole fraction of impurity in the sample.

Influences that decrease the accuracy of this method during a freezing sequence are as follows: (1) Crystallization does not begin as soon as the freezing point is reached since some supercooling is unavoidable. Because of this supercooling, the rise in temperature after the onset of crystallization is not instantaneous and equilibrium temperature values are slowly approached. Since the heat from crystallization is proportional to the amount of solid produced and this amount of solid is in turn approximately proportional to the second power of the supercooling, recovery can never be complete, and the rate of approach to equilibrium becomes progressively slower. A large part of the curve is affected, and the error is particularly serious with very pure samples. (2) As crystallization advances, the total volume of the sample decreases. This decreases the total surface of the sample capable of radiating heat to the surrounding space and causes temperatures during the latter part of the freezing 
experiment to be high. (3) During crystallization some of the solid forms on the wall of the container. Since this is less heated by the release of latent heat, it becomes colder than the rest of the sample. From this effect, at later times in the run, the recorded temperatures will tend to be high. (4) In unstirred systems the distribution of the solid throughout the cell may markedly change the thermal conduction pattern throughout the freezing: Further, in stirred systems, an increasing proportion of solid in the solid-liquid mixture increases the resistance to stirring, causing the stirring energy to increase and the observed temperatures to be high.

When the direction of this procedure is reversed, i.e., when a frozen sample is melted, there are a different group of factors which interfere with the accuracy of results. As we interpret the situation, these principal effects are as follows: (1) The solid, being more dense than its own liquid phase (except for ice and a few other materials) melts loose from the container and settles to the bottom. This is true even for stirred samples when stirring is achieved in the usual fashion. As a consequence, the heat sink created by the latent heat of fusion will be at the bottom of the container where the temperature will not rise above the equilibrium value. However, in the upper parts of the sample there will be little or no solid phase and the temperature here will tend to be higher than the thermodynamic equilibrium temperature for the complete sample. This effect, present from the beginning of the melt, would tend to distort the hyperbolic curve as so to decrease the final estimate of the difference between the actual melting point of the sample and that of the pure material. (2) As a consequence of the segregation of solid and liquid, the composition of the liquid phase is not homogeneous, unless the process is run very slowly. Since the liquid immediately surrounding the pure solid must necessarily be more pure than the bulk liquid, the local equilibrium temperature between solid and liquid in the vicinity of the solid will be high. This effect also would decrease the apparent steepness of the observed melting curve and so decrease the estimate of impurity in the sample. While this difficulty can be minimized by employing extremely slow melting, errors which result from the slower rate now tend to be more significant. (3) Ordinary techniques of stirring are not a feasible means of achieving compositional equilibrium since the solid is originally united in one piece and does not melt so as to fall apart. Inadequate stirring fails to abolish the inhomogeneity mentioned in (2) above.

Most precision calorimeters used for the determination of purity employ a metal vane system to provide temperature equality. Some calorimeters have vertical vanes all directed radially toward the thermometer well whereas others have parallel horizontal vanes normal to the axis of the well. The horizontal vane system would seem to be more suitable in that the vanes tend to keep the solid suspended throughout the cell and in intimate contact with the liquid. With the vertical vanes it is at least con- ceivable that all the solid would be at the bottom and all the liquid in the top portion of the well, making: both thermal and compositional equality difficult to maintain. Even with the horizontal vanes there will be a tendency, during the contractions that accompany freezing, for the liquid to run off the upper vanes leaving the solid out of contact with the liquid during a significant amount of the freezing or melting process.

The principal difference between these vane systems and that described in this paper lies in the construction of the horizontal vanes. Instead of being flat disks, they are pans, arranged on a central support. In operation, each pan holds its own volume of material while maintaining thermal contact with the rest of the system throughout the central support. Now, despite volume changes, each pan represents in miniature what must be taking place in the other pans; each by holding both the solid and liquid phases in thin, horizontal sheets maintains good compositional and thermal contact. We anticipated that the experiments would confirm the attainment of a uniform solid-liquid equilibrium throughout the system during both freeze and subsequent melt.

\section{Apparatus}

In figure 1 is shown a portion of the cell, enough to illustrate the form and use of two of the pans. As represented, the sample is being melted. During the freezing process the sample contracts so that the space between the pans is no longer entirely filled. During the remelting, the liquid flows over the remaining solid, submerging it without anywhere permitting a large separation between the liquid and the solid from which it has formed.

The amount of sample used is such that, when all liquid is at its freezing point, the quantity of sample is just sufficient to cover the top pan. This corresponds to $1 \frac{1}{2} \mathrm{ml}$ of sample in our case. Figure 3 depicts the

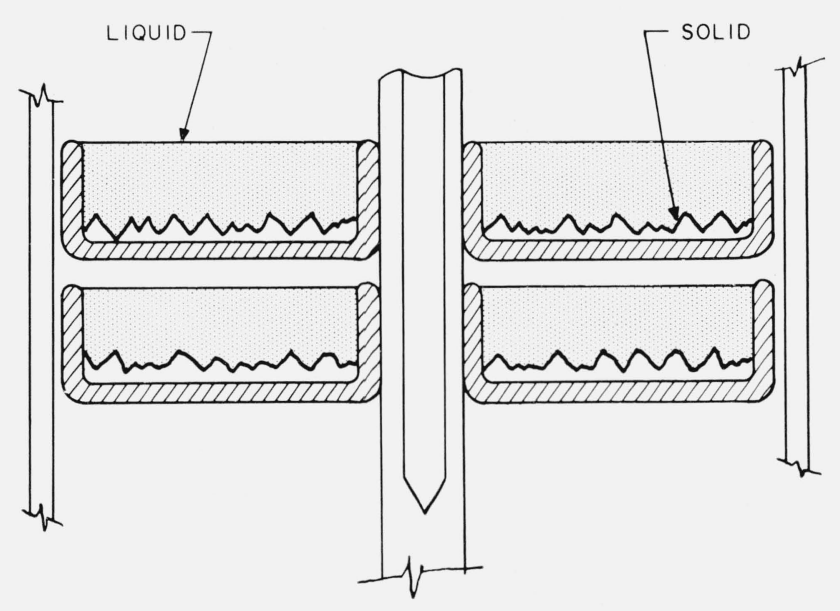

Figure 1. Section of cell showing two pans.

The pans are made from a soft gold sheet and are gold-soldered to the platinum support. 


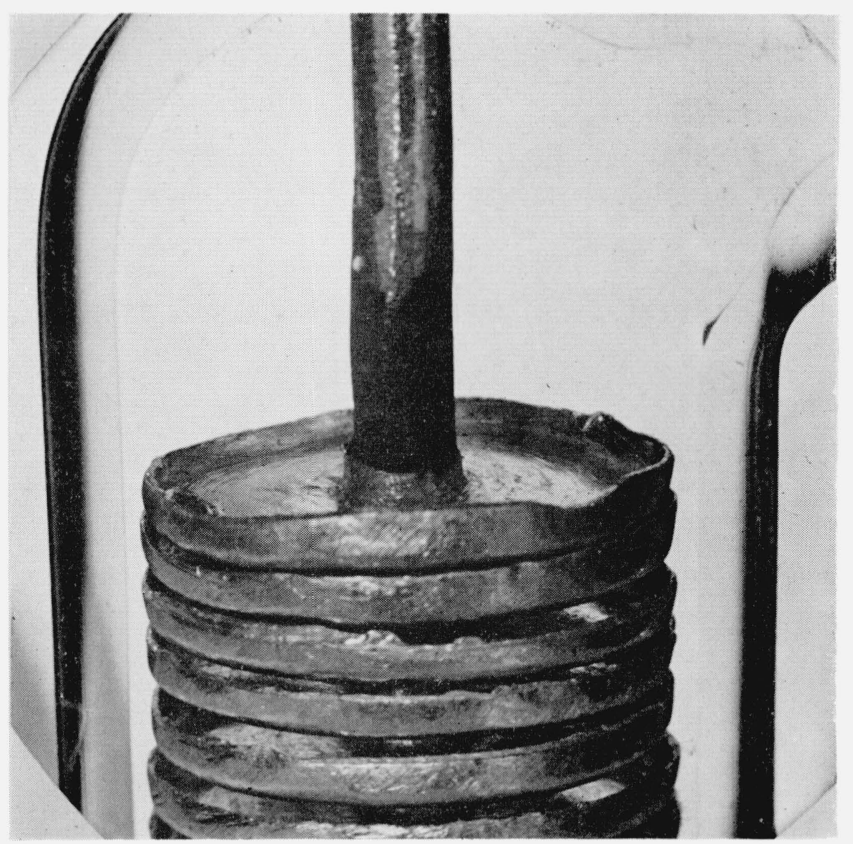

Figure 2. Photomacrograph of cell details, $\times 5.5$.

The cell has been filled with 1,2-dibromoethane (refractive index about 1.54) and immersed in the same liquid. This has limited distortions that would have been caused by the glass envelope (refractive index about 1.51) and significantly increased depth of focus. The inner surface of the glass tube is only dimly shown.

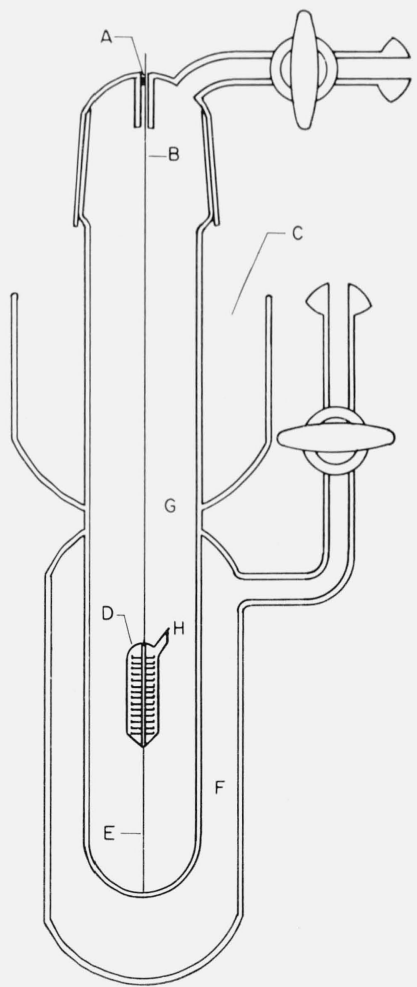

Figure 3. Cryometric cell and its container.

A. Vacuum-tight seal of cell container and thermocouple leads. Seal made with B. Thermocouple leads.

C. Well for thermostating liquid. Used to prevent heat leak down sides of wall. D. The glass envelope of the cell.

E. Glass fiber to hold the cell in place.

E. Glass fiber to hold the
F. Outer vacuum jacket.

F. Outer vacuum jacket.

G. Inner vacuum jacket.
H. Fused seal. Originally point at which cell was connected to transfer manifold. normal assembly of the cell in its container. A 1.5 $\mathrm{mm}$ platinum rod, drilled so as to hold the thermocouple, serves as the support for the pans. These pans are made from 0.0006 in. sheet gold and are gold-soldered to the platinum rod. The rod is sealed into the thin-walled, soft-glass cell as shown. An insulated, copper-constantan thermocouple (No. 36 $\mathrm{B} \& \mathrm{~S}$ wire) is inserted into the platinum support rod, and is secured to the rod with epoxy resin. The only cell supports are the copper and constantan wires, which are sealed at point (A) as they leave the container. The cell is centered and restrained from large oscillations by a thin glass fibre cemented to the bottom of the cell and the bottom of the inner container. All the interior surfaces of the container are silvered, as is the outside of the cell.

A water-ice triple-point cell is used to provide the reference junction temperature, and a thermally and electrically insulated potentiometer is used to balance the junction emf. The signal from the potentiometer is amplified and recorded on a potentiometric, strip-chart recorder. The amplifier and recording system have been previously described [1]. ${ }^{1}$

\section{Experimental Procedure ${ }^{2}$}

The thermocouple was calibrated by comparing the junction emf with the temperature as determined with a calibrated platinum resistance thermometer while both sensing devices were being used to record simultaneously the freezing curve of a $50 \mathrm{ml}$ sample of benzene. The thermometric cell and experimental details of this calibration are described in reference [2].

The cell was then sealed into a glass transfer manifold similar to that described previously [2]. After thorough evacuation of the manifold, a degassed benzene sample was distilled into the cell. The sample was frozen by immersion of the cell in a cold bath, and the cell was removed from the manifold by flame-fusion at $(\mathrm{H})$.

The cell was assembled in its container as shown in figure 2, and the sample was frozen and cooled to $-45{ }^{\circ} \mathrm{C}$. Both of the vacuum jackets were evacuated, and the container was transferred to a water bath whose temperature was held constant. The well (C) was filled with a water-ice mixture to prevent any heat leak down the container wall.

The solid-warming rate was adjusted by varying the pressure in the two vacuum jackets. During the warming of the solid, time versus temperature recordings were made. The melting curves were recorded automatically using the programming sequences described in reference $[2,3]$. One of the melting curves is shown in figure 4.

The end result of the time-temperature melting (freezing) experiment is, of course, the determination of the purity of the sample. The analyst must determine two numbers: $T_{f}$, the freezing point of the particular sample, and $T_{f_{0}}$, the freezing point of the

1 Figures in brackets indicate the literature references at the end of this paper

2 The experimental procedure described herein is specifically that used with the benzene samples but of course could be generalized to apply to any sample. 


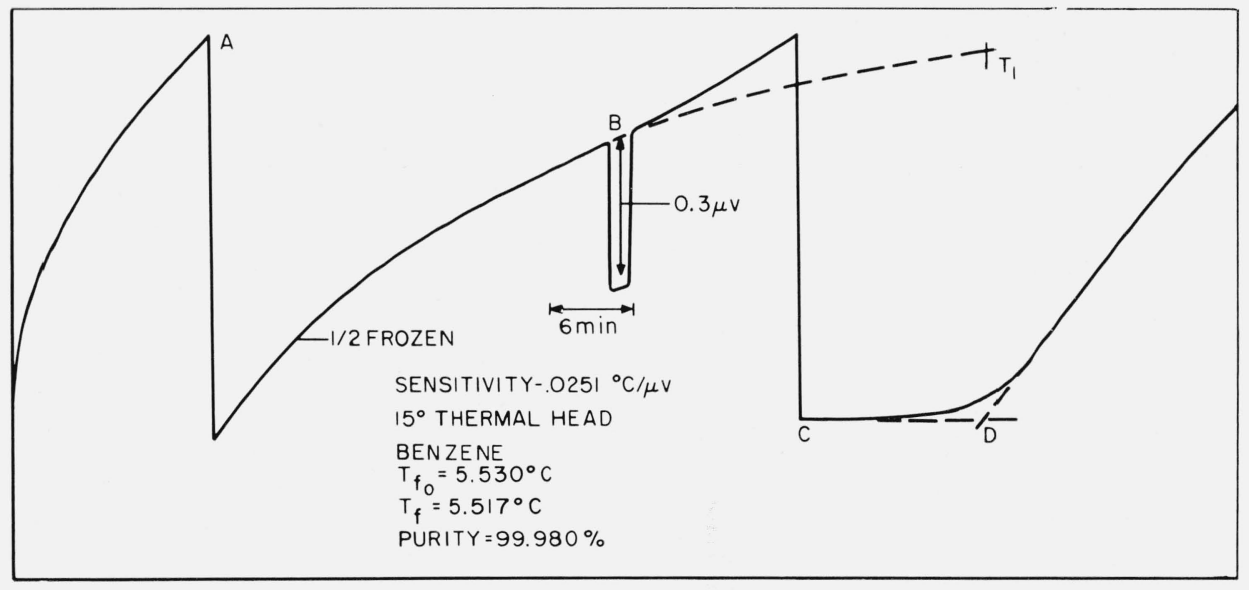

Figure 4. Programmed freezing curve.

A. Position at which zero of recorder was automatically shifted.

B. $0.3 \mu \mathrm{v}$ signal used to determine experimental sensitivity.
C. Automatic reduction of amplification.

D. Intercept of melting curve and liquid warming line,
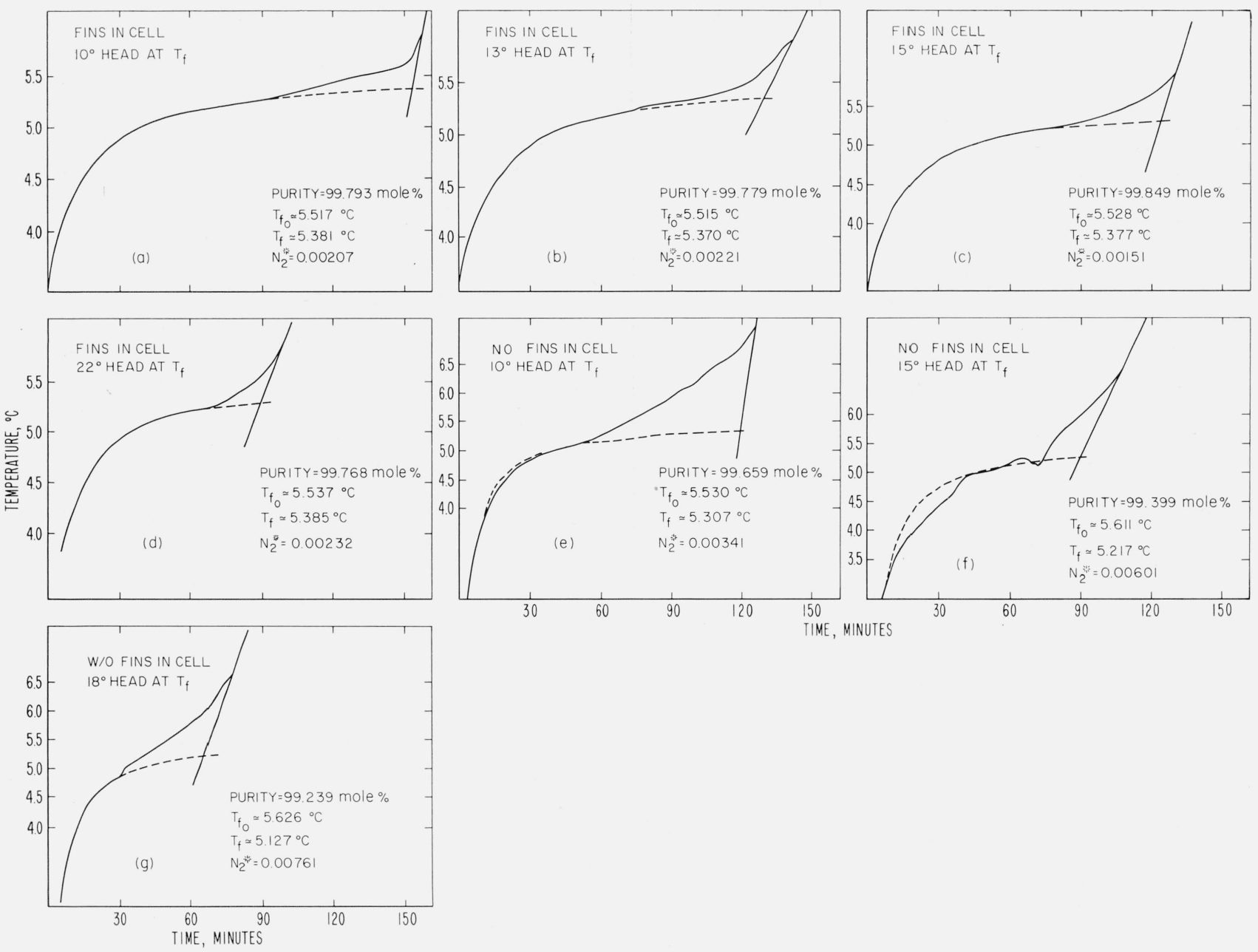

FiguRe 5. Melting curves obtained using the two types of small cell.

Benzene with a known purity of $99.79_{7}$ mole percent was used in both cells. Figures 5 (a,b,c.d) represent experimints conducted in cell with pan-shaped gold fins. Figure $5(\mathrm{e}, \mathrm{f}, \mathrm{g})$ show experiments conducted in a cell without conducting vanes. In these experiments the difference between $T_{f}$ and $T_{f_{0}}$ is much more accurate than their absolute values.

38 
pure material. Under the assumptions made in section 2, the time-temperature melting curve is hyperbolic, where $\mathrm{T}_{f}$ represents the intersection of the hyperbola with the liquid-warming curve and $T_{f_{0}}$ represents the temperature corresponding to the horizontal asymptote of the hyperbola. One of the better ways of analyzing the experimental data lies in the direct comparison of the experimental curve with that of a mathematically exact hyperbola [4]. In this optical projection method, the mathematically exact hyperbola is projected, by means of a photographic enlarger, upon the experimental curve. By increasing or decreasing the magnification and shifting parallel to the coordinates, the best fit is obtained. Sufficient information is projected with the hyperbola so that the analyst can determine both $T_{f}$ and $T_{f_{0}}$. The optical projection technique was used on all the curves included in this paper. The real, experimental curve is shown as a continuous line, whereas the projected hyperbolic function is dotted.

Two differrent samples of benzene were analyzed. In the first sample the purity as determined by the method described herein was compared with the purity as determined by time-temperature analysis using a $50-\mathrm{ml}$ sample $[2,3]$. When the second sample was analyzed a portion of the sample was placed in the small, gold-vaned cell and another identical portion was distilled into an all-glass cell, similar in shape and size. The all-glass cell differed only in that the thermocouple well was of glass and in that there were no conducting vanes of any kind. The construction of this type of cell has been described by Skau [5].

\section{Discussion and Results}

When the first sample was analyzed, two different thermal heads were used, namely 20 and $10 \mathrm{deg}$. The thermal head describes the difference in temperature between the constant-temperature warming bath and the melting point of the sample. The results of these analyzes were, respectively, $99.98_{5}$ and $99.98_{0}$ mole percent. When $50 \mathrm{ml}$ of the sample was analyzed thermometrically [3], the purity was calculated to be $99.97_{6}$ mole percent.

There was available for this work a sample of benzene contaminated with $n$-heptane. In an IUPAC project on purity analysis, the purity of this particular sample was known to be $99.79_{7}$ mole percent. Four runs were made with a portion of this material in the gold-vaned cell, and three runs were made in the similar all-glass cell. A variety of thermal heads was employed. The curves are shown in figure 5 . $T_{f}$ and $T_{f_{0}}$ were independently determined on each curve; the difference between their numbers in each case is more accurate than the absolute value of either. It is evident that with the cell containing the vanes a larger portion of the melt is at temperatures represented by the assumed hyperbola and that the calculated purities correspond much more nearly to the reference value. A tabulation of these results is given in table 1.

We believe that, within the limitations of the test, the gold-vaned cell and apparatus described are capable of approximately the same degree of accuracy as that obtainable from systems employing samples of much larger size. Further, the accuracy which appears to have been obtained with this apparatus is thought to be largely the result of the pan-shaped conducting vanes. We interpret this as a substantiation of the principle of avoiding the transfer of liquid away from the solid from which it has melted. If this is correct, it would seem that the incorporation of the principle into larger adiabatic calorimeters would permit shorter equilibration times and result in the attainment of better thermodynamic equilibrium.

TABle 1. Purity analysis of second benzene sample*

\begin{tabular}{c|c|c|c|c}
\hline \hline Cell a & $\begin{array}{c}\text { Thermal } \\
\text { head }\end{array}$ & $T_{f_{0}}$ & \multicolumn{2}{|c}{ Calculated values } \\
& & & $T_{f}$ & Purity \\
\hline & $\circ C$ & & & Mole \% \\
1 & 10 & 5.517 & 5.381 & 99.793 \\
1 & 13 & 5.515 & 5.370 & 99.779 \\
1 & 15 & 5.528 & 5.377 & 99.849 \\
1 & 22 & 5.537 & 5.385 & 99.768 \\
2 & 10 & 5.530 & 5.307 & 99.659 \\
2 & 15 & 5.611 & 5.217 & 99.399 \\
2 & 18 & 5.626 & 5.127 & 99.239 \\
\hline
\end{tabular}

*Actual purity of sample was known to be 99.797 mole $\%$.

a Cell (1) is vbe gold-vaned one and (2) is the all-glass one.

\section{References}

[1] G. S. Ross and H. D. Dixon, J. Research NBS 64C (Engr. and Instr.) No. 4, 271-275 (1960)

[2] A. R. Glasgow, Jr., G. S. Ross, A. T. Horton, D. Enagonio, H. D. Dixon, C. P. Saylor, G. T. Furukawa, M. L. Reilly, and J. M. Henning, Anal. Chim. Acta 17, 54 (1957).

[3] G. S. Ross and H. D. Dixon, J. Research NBS 67A (Phys. and Chem.) No. 3, 247-251 (1963).

[4] C. P. Saylor, Anal. Chim. Acta 17, 36 (1957).

[5] E. L. Skau, J. Phys. Chem. 37, 609 (1933).

(Paper 68C1-150) 